\title{
STUDYING ONE OF THE PROBLEMS OF WAVE FILTRATION
}

\author{
Michael Bubenchikov ${ }^{1,2}$, Elena Mishcharina ${ }^{2}$, and Eduard Libin ${ }^{2, *}$ \\ ${ }^{1}$ LLC "Gazprom Transgaz Tomsk”, 634029 Tomsk, Russia \\ ${ }^{2}$ National Research Tomsk State University, 634050 Tomsk, Russia
}

\begin{abstract}
Motions of particles through a permeable barrier are studied from the standpoint of Schrodinger wave dynamics. We consider time evolution of a substance concentration spot in the vicinity of one or two potential barriers. To solve the problem we developed a computer technology for integration of the non-stationary Schrödinger equation. The technology is based on symbol mapping and the matrix exponential method which was applied earlier to solve the classical heat equation. The developed theory is relevant to development of nanotechnology, in particular, to studies on permeability of nanoporous membranes.
\end{abstract}

\section{Introduction}

There is a serious problem in finding the coefficient of permeability for an ultra-thin layer of a nanoporous material. If to use methods of classical molecular dynamics [1-15], we risk getting a disproportionately large number of tests for starting molecules with different initial positions and velocities. In application of the Monte Carlo simulation, we are also confronted with a great volume of work associated with statistical processing of information. All this does not guarantee reliable values for the transmission and reflection coefficients. It is possible to introduce into consideration density of a medium being filtered through a nanoporous material. Then potential (elastic) interactions between the medium and the structure will be described by the wave equation. Additionally, if to note that the square of the wave function module is the density, integration of the nanoscopic Schrödinger equation allows finding the density distribution in front of, within and behind the barrier, and in the end, determining the transmission and reflection coefficients. Therefore, in recent years, there has been a number of works devoted to the use of this approach $[16,17]$, which in the future can be successfully applied to study permeability of materials composed of grapheme-like structures [18-24].

\section{Problem statement}

The well-known quantum mechanics [25-27] Schrödinger equation is as follows:

\footnotetext{
*Corresponding author: hudobina@mail2000.ru
} 


$$
\frac{\hbar}{i} \frac{\partial \psi}{\partial t}+U(x, y, z) \psi-\frac{\hbar^{2}}{2 m} \Delta \psi=0
$$
form:

With proper choice of scales for the variables $x$ and $t$, it is also recorded in normalized

$$
\frac{\partial \psi}{\partial t}=i(\Delta-U(x, y, z)) \psi
$$

It is not difficult to note that equation (2) is closely connected with the heat equation of the form:

$$
\frac{\partial \theta}{\partial t}=(\Delta-U(x, y, z)) \theta
$$

The connection between the $\psi$ and $\theta$ functions is that if we know any solution of equation (3) written in the form of: $\theta=\theta(x, y, z, t)$, the associated solution of equation (2) has the form: $\psi=\theta(x, y, z$, it $)$, which is a simple matter of replacing the real-time $t$ by its imaginary value it. Additionally, the function $\psi$ is always a complex value, even if the function $\theta$ is real. The square of the $\psi$ function module in quantum mechanics is interpreted as the probability of particle localization in the elementary volume, and, therefore, the value of $\psi$ is called the probability amplitude.

If to eliminate the time form equation (2), assuming that $\psi=\Psi e^{-i k t}$, for the function $\Psi$ we obtain the stationary Schrödinger equation of the form:

$$
\Delta \psi+\left(k^{2}-U(x, y, z)\right) \psi=0 .
$$

This equation does not contain the time and is managed more easily than equation (2) and it is used mainly for describing passages of particles through a potential barrier or for finding its own values and functions of the operator. The difficulty of analytical or numerical solutions of non-stationary equations (2) or (3) occurs because of the variable coefficient $U(x, y, z)$ present in these equations. Thus, the task is to choose an effective method of solving heat equation (3).

In the one-dimensional case, where the function $\psi$ depends only on one spatial argument $x$, equations (2) and (3) can be written as:

$$
\frac{\partial \psi}{\partial t}=i\left(\frac{\partial^{2}}{\partial x^{2}}-U(x)\right) \psi, \quad \frac{\partial \theta}{\partial t}=\left(\frac{\partial^{2}}{\partial x^{2}}-U(x)\right) \theta .
$$

At any arbitrarily chosen potential barrier $U(x)$, equations (5) can not be solved in an explicit analytic form. Therefore, to determine the general behavioural nature of the solutions, let us consider the case where there is no potential barrier, i.e. where $U(x)=0$ and equations (5) are of the form:

$$
\frac{\partial \psi}{\partial t}=i \frac{\partial^{2} \psi}{\partial x^{2}}, \quad \frac{\partial \theta}{\partial t}=\frac{\partial^{2} \theta}{\partial x^{2}}
$$




\section{Calculations by matrix exponential method}

The solution of the homogeneous heat equation of the form (6) can be formally written in the symbolic form, bearing in mind the expansion of the exponential differential operator in the Taylor series:

$$
\theta(x, t)=e^{t \frac{\partial^{2}}{\partial x^{2}}} \theta(x, 0)=\left[1+t \frac{\partial^{2}}{\partial x^{2}}+\frac{t^{2}}{2 !} \frac{\partial^{4}}{\partial x^{4}}+\frac{t^{3}}{3 !} \frac{\partial^{6}}{\partial x^{6}}+\frac{t^{4}}{4 !} \frac{\partial^{8}}{\partial x^{8}}+\cdots\right] \theta(x, 0) .
$$

Thus, for example, it is not difficult to verify directly that if $\theta(x, 0)$ is the polynomial in the variable $x$, then the row in formula (7) is cut off and we get the exact solution of heat equation (6). On the other hand, in case of numerical implementation of the solution, the differentiation operator with respect to variable $x$ is usually replaced by its finite-difference approximation:

$$
\frac{\partial^{2} \theta}{\partial x^{2}} \approx \frac{\theta_{n-1}-2 \theta_{n}+\theta_{n+1}}{h^{2}}
$$

Thus, the differentiation operator is the three-diagonal square matrix $\mathrm{H}_{2}$ acting on the column vector consisting of numbers $\theta_{n}, n=1,2,3, \ldots N$. In this case, bearing in mind that $\theta$ is the column vectors, we can simply write solution (7) in the vector-matrix form:

$$
\theta(x, t)=\operatorname{expm}\left(t H_{2}\right) \cdot \theta(x, 0) .
$$

The function of the matrix $f(\mathrm{~A})=e^{\mathrm{A}}$ known as the matrix exponential is a standard program in a number of computer systems, and it can be used in solving more general equations (5) which take into account the effect of the potential barrier $U(x)$. It is only necessary to subtract the diagonal matrix $\mathrm{U}\left(x_{i}\right)$ from the differential operator $\mathrm{H}_{2}$. Then we get:

$$
\psi(x, t)=\mathrm{e}^{i t H} \psi(x, 0), \quad \theta(x, t)=\mathrm{e}^{i t H} \theta(x, 0), \quad \mathrm{H}=\mathrm{H}_{2}-\operatorname{diag}(U) .
$$

Let us note the following properties of calculating the Schrödinger equation by the matrix exponential:

1. This method takes into account the presence of potential barriers in the Schrödinger equation, which is practically impossible to implement using analytical methods.

2. Time is included as a parameter directly in the matrix exponential, so formally, integration of the evolutionary coordinate is completed.

3. Calculating the matrix exponential $\mathrm{H}$ at $N<300$ takes a time of about 3-5 seconds. Thus, this method possesses high speed and accuracy.

Automatic execution of the zero boundary condition at the ends of the interval is due to the choice of the matrix $\mathrm{H}_{2}$ as formula (8).

\section{Calculation results}

The figures below show the results of calculations in our program for some cases of potential barriers location in this area of solution. They present a diagram of probability amplitude distribution and its average value over time. 

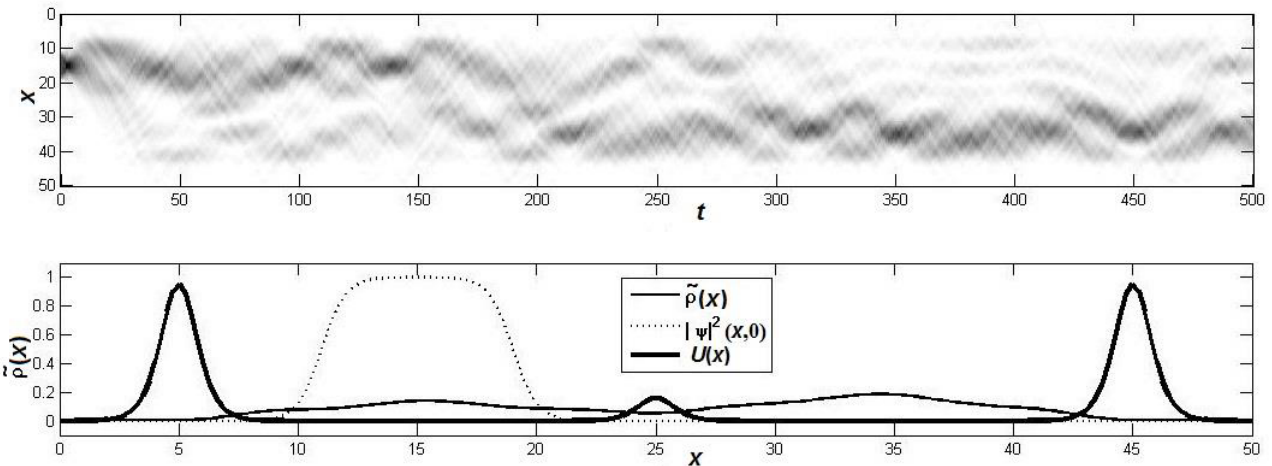

Fig. 1. Probability amplitude distribution and its average value over.
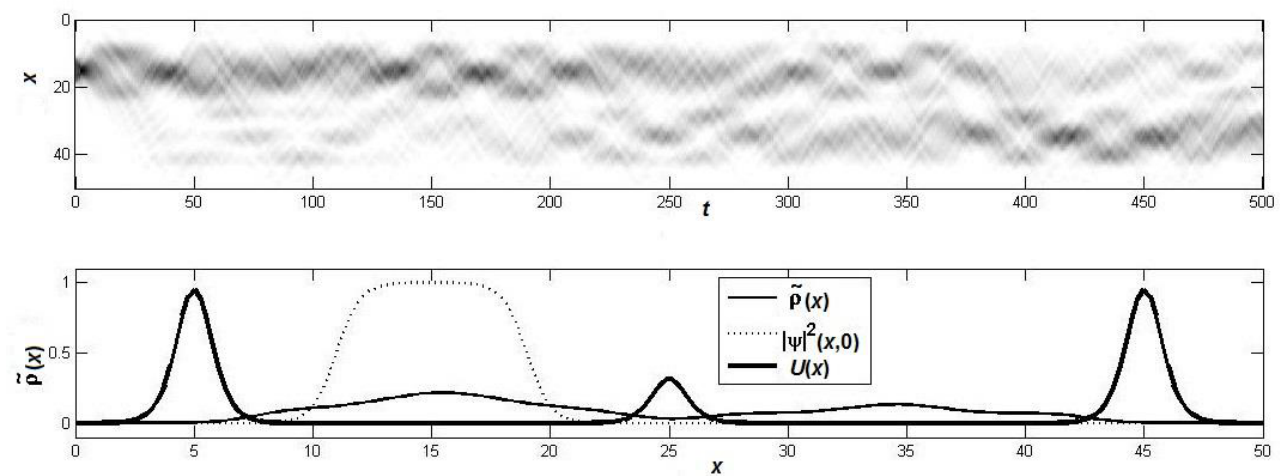

Fig. 2. Probability amplitude distribution and its average value over time.

The dots show the initial distribution of particle density, the solid line indicates the energy of the barriers. We examine permeability of the central barrier which has a significantly smaller size. The peripheral barriers only restrict movement of particles. Initially, the entire mass of particle was located to the left of the central barrier. As can be seen from the average density distribution (fig. 1), more than half the mass went over the barrier. In the second example (fig. 2) the transmission coefficient was lower. Thus, the application of the matrix exponential methods to solving the non-stationary Schrödinger equation showed the expected results when calculating the probability amplitude. This technology can be extended to two-dimensional problems which are of a greater scientific interest with respect to explanations of the absorption phenomenon in such problems.

\section{Acknowledgments}

This paper was written within the frame of Competitiveness Improvement Program of Tomsk State University and with financial support of the Russian Science Foundation, grant under Agreement No. 16-19-00089.

\section{References}

1. V.Ya. Rudyak, S.L. Krasnolutskii, D.A. Ivanov, J. Dok. Phys. 57 (2012)

2. V.Ya. Rudyak, A.A. Belkin, V.V. Egorov, D.A. Ivanov, Int. J. Multiph. 5 (2011)

3. V.Ya. Rudyak, S.L. Krasnolutskii, D.A. Ivanov, J. Microfl. Nanofl. 11 (2011) 
4. V.Ya. Rudyak, S.N. Dubtsov, A.M. Baklanov, Tech. Phys. Let. 34 (2008)

5. V.Ya. Rudyak, S.L. Krasnolutskii, E.N. Ivashchenko, J. Eng. Phys. Thermoph. 81 (2008)

6. V.Ya. Rudyak, J. VDI-Berichte 1803 (2003)

7. V.Ya. Rudyak, A.A. Belkin, Tech. Phys. Lett. 29 (2003)

8. A.I. Potekaev, M.A. Bubenchikov, Russ. Phys. J. 54 (2011)

9. M.A. Bubenchikov, Russ. Phys. J. 54 (2011)

10. M.A. Bubenchikov, A.I. Potekaev, A.M. Bubenchikov, Russ. Phys. J. 56 (2013)

11. M.A. Bubenchikov, A.I. Potekaev, A.M. Bubenchikov, Russ. Phys. J. 56 (2013)

12. A.I. Potekaev, A.M. Bubenchikov, M.A. Bubenchikov, Russ. Phys. J. 55 (2013)

13. M.A. Bubenchikov, A.I. Potekaev, A.M. Bubenchikov, O.V. Usenko, A.V. Ukolov, IOP Conf. Series: Mat. Sc. Eng. 87, 1 (2015)

14. A. M. Bubenchikov, M.A. Bubenchikov, O.V. Matvienko, E. A. Tarasov, O.V. Usenko, AIP Conf. Proc. 1698 (2016)

15. M. A. Bubenchikov, A. M. Bubenchikov, O. V. Usenko, V. A. Poteryaeva, S. Jambaa EPJ Web Conf. 110 (2016)

16. A.M. Bubenchikov, M.A. Bubenchikov, A.I. Potekaev, E.E. Libin, Yu.P. Khudobina, Russ. Phys. J. 57 (2014)

17. A.M. Bubenchikov, M.A. Bubenchikov, A.I. Potekaev, E.E. Libin, Yu.P. Khudobina, Rus. Phys. J. 58 (2015)

18. K.A. Krylova, Y.A. Baimova, S.V. Dmitriev, R.R. Mulyukov, Phys. Sol. St. 58 (2016)

19. A.V. Savin, E.A. Korznikova, S.V. Dmitriev, Phys. Sol. St. 57 (2015)

20. K.A. Bukreeva, R.I. Babicheva, S.V. Dmitriev, K. Zhou, R.R. Mulyukov, A.I. Potekaev, Russ. Phys. J. 57 (2014)

21. J.A. Baimova, B. Liu, S.V. Dmitriev, N. Srikanth, K. Zhou, Phys. Chem. Chem. Phys. $16(2014)$

22. E.A. Korznikova, S.V. Dmitriev, J. Phys. D: Appl. Phys. 47 (2014)

23. E.A. Korznikova, S.Y. Fomin, S .V. Dmitriev, Mat. Sc. Forum 845 (2016)

24. J.A. Baimova, S.V. Dmitriev, Mat. Sc. Forum 838 (2016)

25. H. Feshbach, Ph. M. Morse, Methods of Theoretical Physics. Part II (McGraw-Hill, New York, 1953)

26. L. D. Landau, E. M. Lifshitz: Quantum Mechanics: Non-Relativistic Theory, Vol. 3 (Pergamon Press, Oxford, 1965)

27. J.W.S. Rayleigh, The Theory of Sound, Vol.II (Macmillan, London, 1878) 